\title{
Communication, Collaboration, and Competition
}

\author{
Kathleen C. Taylor \\ MRS President
}

An excitement has spread throughout the materials research community following the discovery of new high temperature superconducting materials. These fascinating developments have caught the attention of the public and have stimulated materials researchers worldwide to a race for the discovery of even higher temperature materials. Some researchers are engaged in research related to the detailed characterization of these materials. Work is under way to fabricate these materials in forms which can take full advantage of the superconducting property.

The 1986 Fall Meeting of the Materials Research Society marked the explosive start of the current search for new high $T_{c}$ superconductors. Presentations and discussions at that meeting during the symposium on Superconducting Materials organized by J. Bevk (AT\&T Bell Laboratories) and A.I. Braginski (Westinghouse Research and Development Center) resulted in researchers returning to their laboratories, stimulated to fabricate the new class of materials they had learned about at this meeting.

Early in January the organizers of the 1987 Spring MRS Meeting (Graham Hubler, Russ Chianelli, and Greg Olson) decided to hold a late news symposium on superconductivity at the MRS Spring Meeting in April to provide a forum for discussion and exchange of the latest technical information coming from laboratories worldwide. This symposium was quickly organized by M. Schluter (AT\&T Bell Laboratories) and D.U. Gubser (Naval Research Laboratory) in the midst of daily news releases which outpaced the usual channels of scientific communication. This symposium, the largest at the meeting, was truly a scientific event, characterized by an intensity which the MRS has developed the flexibility to accommodate.

The visible sense of urgency and excitement which currently pervades the research community working on high $T_{c}$ superconductors is revealing of the way we organize ourselves and interact with one another in the pursuit of R\&D goals. The pace set by this community allows us to examine: (1) the rate limiting step which characterizes our own approach to materials research, (2) the limitations and possibilities present in our working environments for organizing team efforts and for assembling the needed resources and leadership, (3) the responsiveness and flexibility of our national organizations which

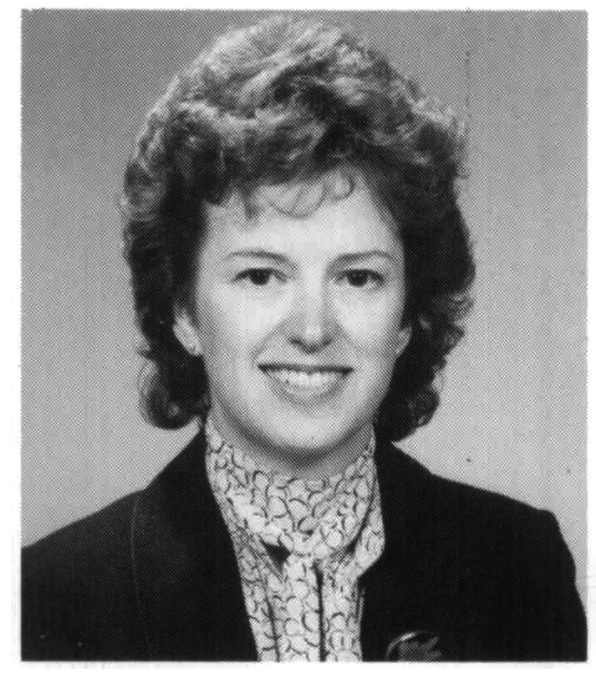

fund materials research, (4) the contradiction of scientific reward systems which aim to stimulate accomplishments through example and competition but may discourage wide collaboration because of the need to identify individual contributions, and (5) our national culture (which I view as a polite way of saying we have little control over our priorities).

The competition among laboratories certainly contributes to the excitement in high $T_{c}$ superconductors. The need for collaboration among researchers is dictated by pace necessary to be in the competition as well as the interdisciplinary effort required to rapidly integrate materials synthesis, materials characterization, and materials processing results with the goal to fabricate useful materials. How collaboration contributes to progress is closely linked to the complementary talents of the team members, a sense of trust and effective communication, the availability and accessibility of resources; and the magnitude and pace of the effort. While a small collaboration can succeed based on the mutual interests and shared efforts of the collaborators alone, a large effort - in particular one aimed to take a new material rapidly from discovery to application in useful products requires structuring. Structuring implies common and ambitious goals, strong and enlightened management to procure and protect the resources, and above all, organization.

In the United States we have achieved preeminence in the past in research which can be done through individual efforts and small collaborations. We know we do not have the lead in taking research findings quickly and economically through devel-

\section{How collaboration contributes to progress is closely linked to the complementary talents of the team members....}

opment to efficient manufacturing. We hear many reasons: lack of management commitment, diversion of resources to correcting mistakes, built-in costs, short-term outlook, not-invented-here syndrome, wrong timing, inadequate technology transfer, etc. I have no doubt that the large effort and teamwork that has characterized successful development of new products offshore can also produce preeminence in research.

I believe we must rapidly change the way we do research - i.e., organize our research groups and communicate our findings - if we are to continue as leaders in research and compete as producers. We must examine the location of the boundaries of our research groups, create collaborations across disciplinary lines, and organize collaborations which are deliberately structured to achieve goals which go beyond communication to our peers. We must instill a greater sense of urgency in our efforts and understand that a relationship exists between output and accomplishment as well as talent and accomplishment.

Let me conclude with some observations I made at the recent symposium on high $\mathrm{T}_{c}$ superconductors at the MRS Spring Meeting. One foreign participant seated in the front row diligently photographed every viewgraph displayed during the fast-paced 18-hour symposium. The group from the University of Tokyo distributed 1,000 copies (air freighted from Japan to the MRS meeting) of a recent issue of the Japanese Journal of Applied Physics featuring a special section of high $\mathrm{T}_{\mathrm{c}}$ oxide superconductors in exchange for the participants supplying their names, addresses, affiliations, and fields of interest. The 84 papers in this issue were received between February 16 and March 18; all were accepted on March 23, the editors having set aside the traditional peer review rule. Surely false pride should never prevent us from profiting from the example of others.

MRS 


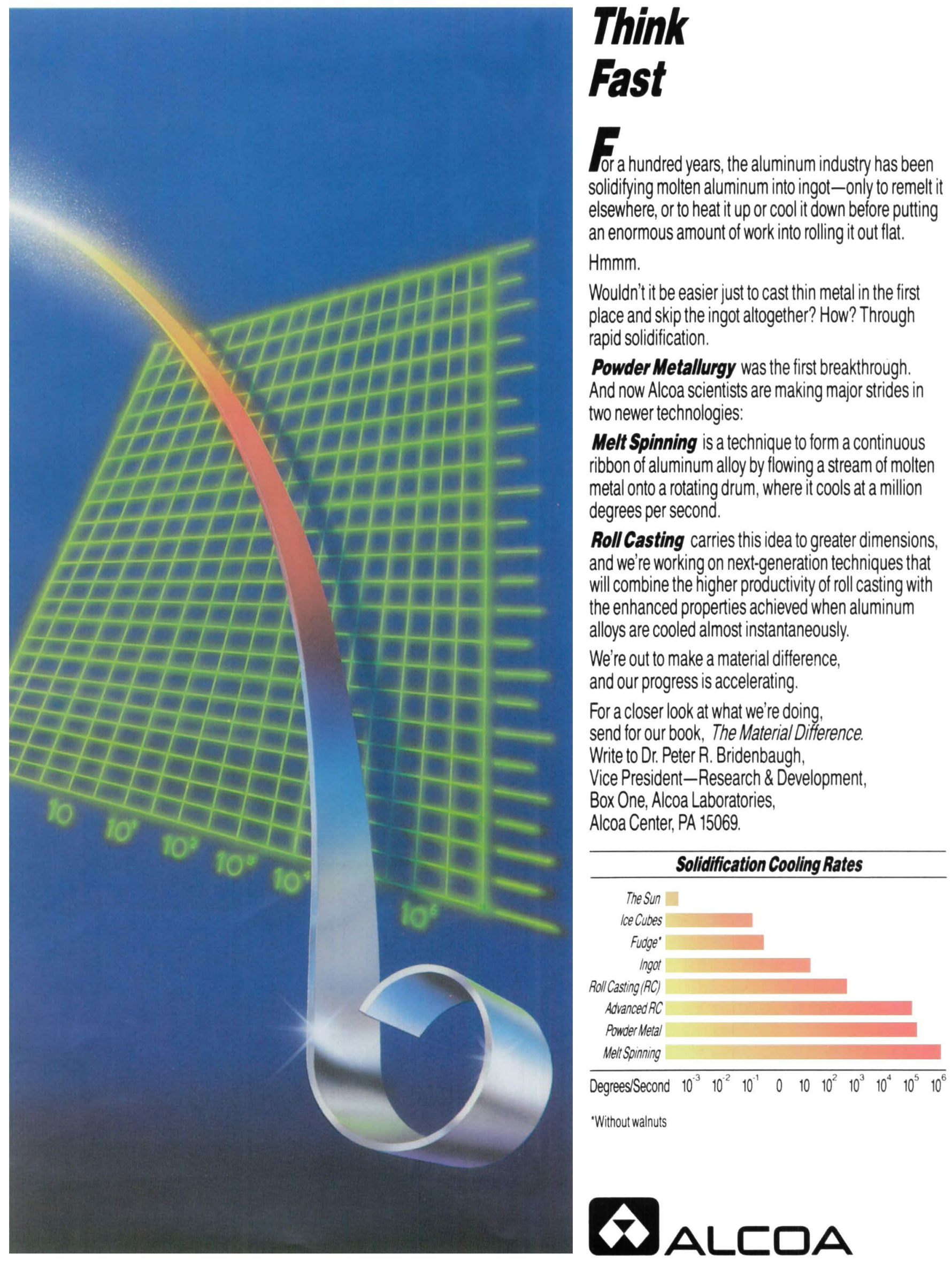

